\title{
Research Article \\ Krein Space Quantization of Casimir Effect for a Spherical Shell
}

\author{
F. Payandeh ${ }^{1,2}$ \\ ${ }^{1}$ Department of Physics, Payame Noor University, P.O. Box 19395-3697, Tehran, Iran \\ ${ }^{2}$ Amirkabir University of Technology, Tehran 15914, Iran \\ Correspondence should be addressed to F. Payandeh, farrin_payandeh@yahoo.com
}

Received 22 August 2011; Accepted 18 September 2011

Academic Editors: S. Paganis and I. V. Vancea

Copyright (C) 2012 F. Payandeh. This is an open access article distributed under the Creative Commons Attribution License, which permits unrestricted use, distribution, and reproduction in any medium, provided the original work is properly cited.

The Casimir stress on a spherical shell in de Sitter spacetime for a massless scalar field is calculated using Krein space quantization. In this method, the auxiliary negative frequency states have been utilized, the modes of which do not interact with the physical states and are not affected by the physical boundary conditions. These unphysical states just play the role of an automatic renormalization tool for the theory.

\section{Introduction}

The Casimir effect is a small attractive force acting between two parallel uncharged conducting plates and it is regarded as one of the most striking manifestation of vacuum fluctuations in quantum field theory. It is due to the quantum vacuum fluctuation of the field operator between two parallel plates. In other words, the Casimir effect can be viewed as the polarization of the vacuum by boundary conditions or geometry. The presence of reflecting boundaries alters the zero-point modes of a quantized field and results in the shifts in the vacuum expectation values of quantities quadratic in the field, such as the energy density and stresses.

In particular, vacuum forces arise acting on the constraining boundaries. The particular features of these forces depend on the nature of the quantum field, the type of spacetime manifold and its dimensionality, the boundary geometries, and the specific boundary conditions imposed on the field. Since the original work by Casimir in 1948 [1], many theoretical and experimental works have been done on this problem [2-14].

The time dependence of boundary conditions or geometries, the so-called dynamical Casimir effect, is also a new element which has to be taken into account. In particular, in [15] 
the Casimir effect has been calculated for a massless scalar field satisfying Dirichlet boundary conditions on the spherical shell in de Sitter space. The Casimir stress is calculated for inside and outside of the shell with different backgrounds corresponding to different cosmological constants.

It should be remarked that the procedures of renormalization and regularization have been applied for eliminating the divergences appeared in physical quantities [16-20]. The Casimir effect has been also investigated for two parallel plates in Krein space quantization [21].

The historical background of Krein space quantization goes back to the covariant quantization of minimally coupled scaler field in de Sitter spacetime. It has been shown that the linear quantum gravity in the background field method is perturbatively nonrenormalizable and also there appears an infrared divergence. This infrared divergence does not manifest itself in the quadratic part of the effective action in the one-loop approximation. This means that the pathological behavior of the graviton propagator may be gauge dependent and so should not appear in an effective way as a physical quantity [22]. The infrared divergence which appears in the linear gravity in de Sitter space is the same as the minimally coupled scalar field in de Sitter space [23-27]. It is shown that one cannot construct a covariant quantization of the minimally coupled scalar field with only positive norm states [28]. It has been proved that the use of the two sets of solutions (positive and negative norm states) is an unavoidable feature if one wants to preserve causality (locality), covariance, and elimination of the infrared divergence in quantum field theory for the minimally coupled scalar field in de Sitter space $[29,30]$, that is, Krein space quantization.

The singular behavior of Green's function at short relative distances (ultraviolet divergence) or in the large relative distances (infrared divergence) leads to main divergences in the quantum field theory. It was conjectured that quantum metric fluctuations might smear out the singularities of Green's functions on the light cone, but it does not remove other ultraviolet divergences [31]. However, it has been shown that quantization in Krein space removes all ultraviolet divergences of quantum field theory (QFT) except the light cone singularity [32]. By using the Krein space quantization and the quantum metric fluctuations in the linear approximation, it has been showed that the infinities in the Green's function are disappeared [31, 33].

Quantization in Krein space instead of Hilbert space has some interesting features. For example, in this method, the vacuum energy becomes zero naturally, so the normal ordering would not be necessary $[29,32]$. The auxiliary negative norm states, which are used in the Krein space quantization, play the regularization of the theory.

Applying the unphysical negative frequency states and defining the field operator in Krein space, we can calculate the gravitational pressure on a spherical shell yielding the standard result obtained.

\section{Scalar Casimir Effect for a Sphere in de Sitter Space}

The Casimir force due to fluctuations of a free massless scalar field satisfying Dirichlet boundary conditions on a spherical shell in Minkowski space time has been studied in [34]. Doing the calculations in Krein space, the two-point Green's function $G_{K}\left(x, t ; x^{\prime}, t^{\prime}\right)$ is defined 
as the vacuum expectation value of the time-ordered product of two fields ( $K$ and $T$ stand for quantities in Krein space and the time-ordered product, resp.)

$$
\begin{gathered}
G_{K}\left(x, t ; x^{\prime}, t^{\prime}\right) \equiv-i\left\langle 0\left|T \Phi_{K}(x, t) \Phi_{K}\left(x^{\prime}, t^{\prime}\right)\right| 0\right\rangle, \\
\Phi_{K}(x, t)=\sum_{\vec{k}}\left[\left(a_{\vec{k}}+b_{\vec{k}}^{\dagger}\right) u_{p}(k, x)+\left(a_{\vec{k}}^{\dagger}+b_{\vec{k}}\right) u_{n}(k, x)\right] .
\end{gathered}
$$

The operators $a^{\dagger}(\vec{k})$ and $a(\vec{k})$ create and destroy, respectively, the mode $u_{p}(k, x)$ with positive energy $\left(k^{0}=\omega_{\vec{k}}\right)$, which may be considered as the operators of creation and annihilation of a particle and the operators $b^{\dagger}(\vec{k})$ and $b(\vec{k})$ create and destroy, respectively, the mode $u_{n}(k, x)$ with negative energy $\left(-k^{0}=-\omega_{\vec{k}}\right)$, which may be considered as the operators of creation and annihilation of an "antiparticle" in the inverse time direction. The two sets of modes do not affect each other and in the standard QFT, the negative energy states are eliminated in the quantum field operators, which are the origin of the appearance of divergence. On the contrary, the divergence disappears by taking these states into account. The two-point Green's function has to satisfy the Dirichlet boundary conditions on the shell:

$$
\left.G_{K}\left(x, t ; x^{\prime}, t^{\prime}\right)\right|_{|x|=a}=0,
$$

where $a$ is radius of the spherical shell. The stress-energy tensor in Krein space $T_{K}^{\mu v}(x, t)$ is given by

$$
\begin{aligned}
T_{K}^{\mu \nu}(x, t) \equiv & \partial^{\mu} \Phi_{K}(x, t) \partial^{v} \Phi_{K}(x, t) \\
& -\frac{1}{2} \eta^{\mu v} \partial_{\curlywedge} \Phi_{K}(x, t) \partial^{\lambda} \Phi_{K}(x, t)
\end{aligned}
$$

The radial Casimir force per unit area $F / A$ on the sphere, called Casimir stress, is obtained from the radial-radial component of the vacuum expectation value of the stressenergy tensor:

$$
\frac{F}{A}=\left.\left\langle 0\left|T_{\text {in }}^{r r}-T_{\text {out }}^{r r}\right| 0\right\rangle\right|_{r=a}
$$

Taking into account the relation (2.1) between the vacuum expectation value of the stress-energy tensor $T_{K}^{\mu \nu}(x, t)$ and the Green's function at equal times $G_{K}\left(x, t ; x^{\prime}, t\right)$, we obtain

$$
\begin{aligned}
\frac{F}{A}=\frac{i}{2}[ & \frac{\partial}{\partial r} \frac{\partial}{\partial r^{\prime}} G_{K}\left(x, t ; x^{\prime}, t\right)_{\mathrm{in}} \\
& \left.-\frac{\partial}{\partial r} \frac{\partial}{\partial r^{\prime}} G_{K}\left(x, t ; x^{\prime}, t\right)_{\text {out }}\right]\left.\right|_{x=x^{\prime},|x|=a} .
\end{aligned}
$$


One may use of the above flat space calculation in de Sitter space time by taking the de Sitter metric in conformally flat form

$$
d s^{2}=\Omega(\eta)\left[d \eta^{2}-\sum_{l=1}^{3}\left(d x^{1}\right)^{2}\right]
$$

where $\Omega(\eta)=\alpha / \eta$ and $\eta$ is the conformal time

$$
-\infty<\eta<0
$$

Assuming a canonical quantization of the scalar field in Krein space, the conformally transformed quantized scalar field in de Sitter spacetime is given by

$$
\bar{\Phi}_{K}(x, \eta)=\sum_{k}\left[\left(a_{\vec{k}}+b_{\vec{k}}^{\dagger}\right) \bar{u}_{k}(\eta, x)+\left(a_{\vec{k}}^{\dagger}+b_{\vec{k}}\right) \bar{u}_{k}^{*}(\eta, x)\right]
$$

where $a_{k}^{\dagger}$ and $a_{k}$ are creation and annihilation operators, respectively, and the vacuum states associated with the physical modes $\bar{u}_{k}$ defined by $a_{k}|\overline{0}\rangle=0$ are called conformal vacuum. Given the flat space Green's function (2.1), we obtain

$$
\bar{G}_{K}=-i\left\langle\overline{0}\left|T \bar{\Phi}_{K}(x, \eta) \bar{\Phi}_{K}\left(x^{\prime}, \eta^{\prime}\right)\right| \overline{0}\right\rangle=\Omega^{-1}(\eta) \Omega^{-1}\left(\eta^{\prime}\right) G_{K},
$$

where $\bar{\Phi}_{K}(x, \eta)=\Omega^{-1}(\eta) \Phi_{K}(x, \eta)$ has been used. Therefore, using (2.5), (2.6), and (2.10), we obtain the total stress on the sphere in de Sitter spacetime and using Krein space quantization as

$$
\frac{\bar{F}}{A}=\frac{\eta^{2}}{\alpha^{2}} \frac{F}{A}
$$

in accordance with the standard result.

\section{Spherical Shell with Different Vacua in Krein Space Quantization}

We assume different vacua inside and outside, corresponding to different $\alpha_{\text {in }}$ and $\alpha_{\text {out }}$ for the Lorentzian metric (2.7) and use the following relation for the stress on the shell due to boundary conditions in flat spacetime [8]:

$$
\frac{F}{A}=\frac{-1}{4 \pi a^{2}} \frac{\partial E}{\partial a}
$$

where the Casimir energy $E$ is the sum of Casimir energies $E_{\text {in }}$ and $E_{\text {out }}$ for inside and outside of the shell. The corresponding relation in de Sitter spacetime and applying Krein method leads to the following result [15]:

$$
\frac{\bar{F}}{A}=\frac{-1}{4 \pi a^{2}} \frac{\partial \bar{E}}{\partial a}=\frac{\eta^{2}}{8 \pi a^{4}}\left(\frac{c_{1}}{\alpha_{\text {in }}^{2}}+\frac{c_{2}}{\alpha_{\text {out }}^{2}}\right),
$$


where we have used the automatically renormalized total zero-point energy in Krein Space

$$
\bar{E}=\frac{\eta^{2}}{2 a}\left(\frac{c_{1}}{\alpha_{\text {in }}^{2}}+\frac{c_{2}}{\alpha_{\text {out }}^{2}}\right),
$$

in which $c_{1}=0.008873, c_{2}=-0.003234$.

Now, we obtain the pure effect of vacuum polarization due to the gravitational field without any boundary conditions in Euclidean (outside) region with the following metric:

$$
d s^{2}=-\Omega(\eta)\left[d \eta^{2}+\sum_{l=1}^{3}\left(d x^{i}\right)^{2}\right]
$$

To this end, we calculate the automatically renormalized stress tensor in Krein space quantization for the massless scalar field in de Sitter spacetime with Euclidean signature. One may use [2]

$$
\begin{aligned}
\left.\left\langle 0\left|T_{\mu}^{v}\left[g_{k l}\right]\right| 0\right\rangle\right|_{K}= & \left.\left(\frac{\tilde{g}}{g}\right)^{1 / 2}\left\langle 0\left|T_{\mu}^{v}\left[\widetilde{g}_{k l}\right]\right| 0\right\rangle\right|_{K} \\
& -\frac{1}{2880 \pi^{2}}\left[\frac{1}{6}{ }^{(1)} H_{\mu}^{v}-{ }^{(3)} H_{\mu}^{v}\right],
\end{aligned}
$$

where $\tilde{g}_{k l}$ is the flat Euclidean metric for which $\left.\left\langle 0\left|T_{\mu}^{v}\left[\tilde{g}_{k l}\right]\right| 0\right\rangle\right|_{K}=0$, and

$$
\begin{gathered}
{ }^{(1)} H_{\mu}^{v}=0, \\
{ }^{(3)} H_{\mu}^{v}=\frac{3}{\alpha^{4}} \delta_{\mu}^{v} .
\end{gathered}
$$

We then obtain

$$
\left.\left\langle 0\left|T_{\mu}^{v}\left[g_{k l}\right]\right| 0\right\rangle\right|_{K}=\frac{1}{960 \pi^{2} \alpha^{4}} \delta_{\mu^{\prime}}^{v}
$$

which is exactly the same result for the Lorentzian case [2]. Therefore, the corresponding effective radial pressures for the Euclidean (outside) and Lorentzian (inside) regions with $\alpha_{\text {out }}$ and $\alpha_{\text {in, }}$, due to pure effect of gravitational vacuum polarization without any boundary condition, are given, respectively, by

$$
\begin{gathered}
P_{\text {out }}^{E}=-\left.\left\langle 0\left|T_{r}^{r}\left[g_{k l}\right]\right| 0\right\rangle\right|_{K}=-\frac{1}{960 \pi^{2} \alpha_{\text {out }}^{4}}, \\
P_{\text {in }}^{L}=-\left.\left\langle 0\left|T_{r}^{r}\left[g_{k l}\right]\right| 0\right\rangle\right|_{K}=-\frac{1}{960 \pi^{2} \alpha_{\text {in }}^{4}} .
\end{gathered}
$$


The corresponding gravitational pressure on the spherical shell in Krein space quantization is then given by

$$
P_{G}=P_{\text {in }}^{L}-P_{\text {out }}^{E}=-\frac{1}{960 \pi^{2}}\left(\frac{1}{\alpha_{\text {in }}^{4}}-\frac{1}{\alpha_{\text {out }}^{4}}\right) .
$$

\section{Conclusion}

The Casimir effect has been studied for spherical bubbles with different vacua inside and outside, corresponding to de Sitter metrics. The metrics inside and outside are taken by Lorentzian and Euclidean, respectively. The case of different vacua in a Lorentzian de Sitter spacetime has already been studied in [15]. In the present work, we have shown that the same results could be attained using Krein space quantization. Presence of the unphysical negative frequency states plays the role of an automatic renormalization tool.

\section{Appendix}

In this appendix, we review the elementary facts about Krein space quantization. As we know, the origin of divergences in standard quantum field theory lies in the singularity of the Green's function. The divergence appears in the imaginary part of the Feynman propagator, and the real part is convergent [35]:

$$
\begin{aligned}
G_{F}^{P}\left(x, x^{\prime}\right)= & -\frac{1}{8 \pi} \delta\left(\sigma_{0}\right) \\
& +\frac{m^{2}}{8 \pi} \theta\left(\sigma_{0}\right)\left[\frac{J_{1}\left(\sqrt{2 m^{2} \sigma_{0}}\right)-i N_{1}\left(\sqrt{2 m^{2} \sigma_{0}}\right)}{\sqrt{2 m^{2} \sigma_{0}}}\right] \\
& -\frac{i m^{2}}{4 \pi^{2}} \theta\left(-\sigma_{0}\right) \frac{K_{1}\left(\sqrt{2 m^{2}\left(-\sigma_{0}\right)}\right)}{\sqrt{2 m^{2}\left(-\sigma_{0}\right)}} .
\end{aligned}
$$

Consideration of negative frequency states removes singularity of the Green's function with exception of delta function singularity:

$$
\begin{aligned}
G_{T}\left(x, x^{\prime}\right)= & -\frac{1}{8 \pi} \delta\left(\sigma_{0}\right) \\
& +\frac{m^{2}}{8 \pi} \theta\left(\sigma_{0}\right) \frac{J_{1}\left(\sqrt{2 m^{2} \sigma_{0}}\right)}{\sqrt{2 m^{2} \sigma_{0}}}, \quad \sigma_{0} \geq 0 .
\end{aligned}
$$


However, considering the quantum metric fluctuations removes the latter singularity:

$$
\begin{aligned}
\left\langle G_{T}\left(x, x^{\prime}\right)\right\rangle= & -\frac{1}{8 \pi} \sqrt{\frac{\pi}{2\left\langle\sigma_{1}^{2}\right\rangle}} \exp \left(-\frac{\sigma_{0}^{2}}{2\left\langle\sigma_{1}^{2}\right\rangle}\right) \\
& +\frac{m^{2}}{8 \pi} \theta\left(\sigma_{0}\right) \frac{J_{1}\left(\sqrt{2 m^{2} \sigma_{0}}\right)}{\sqrt{2 m^{2} \sigma_{0}}}
\end{aligned}
$$

where $\left\langle\sigma_{1}^{2}\right\rangle$ is related to the density of gravitons. When $\sigma_{0}=0$, due to the metric quantum fluctuation $\left\langle\sigma_{1}^{2}\right\rangle \neq 0$, and we have

$$
\left\langle G_{T}(0)\right\rangle=-\frac{1}{8 \pi} \sqrt{\frac{\pi}{2\left\langle\sigma_{1}^{2}\right\rangle}}+\frac{m^{2}}{16 \pi} .
$$

By using the Fourier transformation, we obtain [36]

$$
\left\langle\widetilde{G}_{T}(p)\right\rangle=\widetilde{G}_{T}(p)+P P \frac{m^{2}}{p^{2}\left(p^{2}-m^{2}\right)}
$$

However, in the one-loop approximation, the contribution of delta function is negligible and the Green's function in Krein space quantization appearing in the transition amplitude is

$$
\left.\left.\left\langle\widetilde{G}_{T}(p)\right\rangle\right|_{\text {one-loop }} \equiv \widetilde{G}_{T}(p)\right|_{\text {one-loop }} \equiv P P \frac{m^{2}}{p^{2}\left(p^{2}-m^{2}\right)}
$$

\section{Acknowledgment}

The author would like to thank M. V. Takook for useful discussions.

\section{References}

[1] H. B. G. Casimir, “On the attraction between two perfectly conducting plates," Proceedings of the Royal Netherlands Academy of Arts and Sciences, vol. 51, pp. 793-795, 1948.

[2] N. D. Birrel and P. C. W. Davies, Quantum Fields in Curved Space, Cambridge University Press, Cambridge, UK, 1982.

[3] V. M. Mostepanenko and N. N. Trunov, The Casimir Effect and Its Applications, Clarendon, Oxford, UK, 1997.

[4] G. Plunien, B. Müller, and W. Greiner, "The Casimir effect," Physics Reports, vol. 134, no. 2-3, pp. 87-193, 1986.

[5] S. K. Lamoreaux, "Resource letter CF-1: Casimir force," American Journal of Physics, vol. 67, no. 10, pp. 850-861, 1999.

[6] M. Bordag, U. Mohideen, and V. M. Mostepanenko, "New developments in the Casimir effect," Physics Report, vol. 353, no. 1-3, pp. 1-205, 2001.

[7] K. Kirsten, Spectral Functions in Mathematics and Physics, CRC Press, Boca Raton, Fla, USA, 2001.

[8] K. A. Milton, L. L. DeRaad, and J. Schwinger, "Casimir self-stress on a perfectly conducting spherical shell," Annals of Physics, vol. 115, no. 2, pp. 388-403, 1978. 
[9] M. R. Setare and A. Saharian, "Casimir effect in background of static domain wall," International Journal of Modern Physics A, vol. 16, no. 8, pp. 1463-1469, 2001.

[10] M. R. Setare, "The Casimir effect, Hawking radiation and the trace anomaly," Classical and Quantum Gravity, vol. 18, no. 11, p. 2097, 2001.

[11] M. R. Setare and R. Mansouri, "Casimir stress on parallel plates in a de Sitter space," Classical and Quantum Gravity, vol. 18, no. 14, pp. 2659-2663, 2001.

[12] M. R. Setare, "Casimir stress for concentric spheres in de Sitter space," Classical and Quantum Gravity, vol. 18, no. 22, pp. 4823-4829, 2001.

[13] A. A. Saharian and M. R. Setare, "The Casimir effect on background of conformally flat brane-world geometries," Physics Letters B, vol. 552, no. 3-4, pp. 119-126, 2003.

[14] A. A. Saharian and M. R. Setare, "Casimir energy-momentum tensor for a brane in de Sitter spacetime," Physics Letters B, vol. 584, no. 3-4, pp. 306-314, 2004.

[15] M. R. Setare and R. Mansouri, "Casimir effect for a spherical shell in de Sitter space," Classical and Quantum Gravity, vol. 18, no. 12, pp. 2331-2338, 2001.

[16] F. Payandeh, M. Mehrafarin, S. Rouhani, and M. V. Takook, "Free fields quantization in the "Krein" space," Ukrainian Journal of Physics, vol. 53, no. 12, pp. 1202-1207, 2008.

[17] F. Payandeh, "Spinor field in generalized Krein space quantization," Revista Cubana de Física, vol. 26, no. 2B, pp. 232-237, 2009.

[18] F. Payandeh, M. Mehrafarin, and M. V. Takook, "Field quantization in Krein space," in Proceedings of the 13th International Symposium on Particles, Strings and Cosmology (PASCOS '07), vol. 957 of AIP Conference Proceedings, pp. 249-252, July 2007.

[19] F. Payandeh, "Krein space quantization of linear gravity in de Sitter spacetime and generalization to QFT," Journal of Physics: Conference Series, vol. 174, no. 1, Article ID 012056, 2009.

[20] F. Payandeh, M. Mehrafarin, and M. V. Takook, "One-loop approximation of Møller scattering in generalized Krein-space quantization," Science in China, Series G, vol. 52, no. 2, pp. 212-217, 2009.

[21] H. Khosravi, M. Naseri, S. Rouhani, and M. V. Takook, "Casimir effect in Krein space quantization," Physics Letters B, vol. 640, no. 1-2, pp. 48-51, 2006.

[22] I. Antoniadis, J. Iliopoulos, and T. N. Tomaras, "One-loop effective action around De Sitter space," Nuclear Physics B, vol. 462, no. 2-3, pp. 437-452, 1996.

[23] T. Garidi, J. P. Gazeau, S. Rouhani, and M. V. Takook, "“massless" vector field in de Sitter universe," Journal of Mathematical Physics, vol. 49, no. 3, Article ID 032501, 2008.

[24] T. Garidi, J. P. Gazeau, and M. V. Takook, "“Massive” spin-2 field in de Sitter space," Journal of Mathematical Physics, vol. 44, no. 9, pp. 3838-3862, 2003.

[25] S. Behroozi, S. Rouhani, M. V. Takook, and M. R. Tanhayi, "Conformally invariant wave equations and massless fields in de Sitter spacetime," Physical Review D, vol. 74, no. 12, Article ID 124014, 2006.

[26] M. Dehghani, S. Rouhani, M. V. Takook, and M. R. Tanhayi, "Conformally invariant "massless" spin-2 field in the de Sitter universe," Physical Review D, vol. 77, no. 6, Article ID 064028, 2008.

[27] M. V. Takook, M. R. Tanhayi, and S. Fatemi, "Conformal linear gravity in de Sitter space," Journal of Mathematical Physics, vol. 51, no. 3, Article ID 032503, 8 pages, 2010.

[28] B. Allen, "Vacuum states in de Sitter space," Physical Review D, vol. 32, no. 12, pp. 3136-3149, 1985.

[29] J. P. Gazeau, J. Renaud, and M. V. Takook, "Gupta-Bleuler quantization for minimally coupled scalar fields in de Sitter space," Classical and Quantum Gravity, vol. 17, no. 6, pp. 1415-1434, 2000.

[30] M. V. Takook, "Covariant two-point function for minimally coupled scalar field in de Sitter spacetime," Modern Physics Letters A, vol. 16, no. 26, pp. 1691-1697, 2001.

[31] H. L. Ford, “Quantum field theory in curved spacetime," http://arxiv.org/abs/gr-qc/9707062.

[32] M. V. Takook, "Negative norm states in de Sitter space and QFT without renormalization procedure," International Journal of Modern Physics E, vol. 11, no. 6, pp. 509-518, 2002.

[33] S. Rouhani and M. V. Takook, "A naturally renormalized quantum field theory," International Journal of Theoretical Physics, vol. 48, no. 10, pp. 2740-2747, 2009.

[34] C. M. Bender and K. A. Milton, "Scalar Casimir effect for a D-dimensional sphere," Physical Review D, vol. 50, no. 10, pp. 6547-6555, 1994.

[35] F. Payandeh, "Is it possible to find a probable solution for the problem of divergence in QFT?" Journal of Physics: Conference Series, vol. 306, no. 1, Article ID 012054, 2011.

[36] A. Refaei and M. V. Takook, "Scalar effective action in Krein space quantization," Modern Physics Letters A, vol. 26, no. 1, pp. 31-41, 2011. 

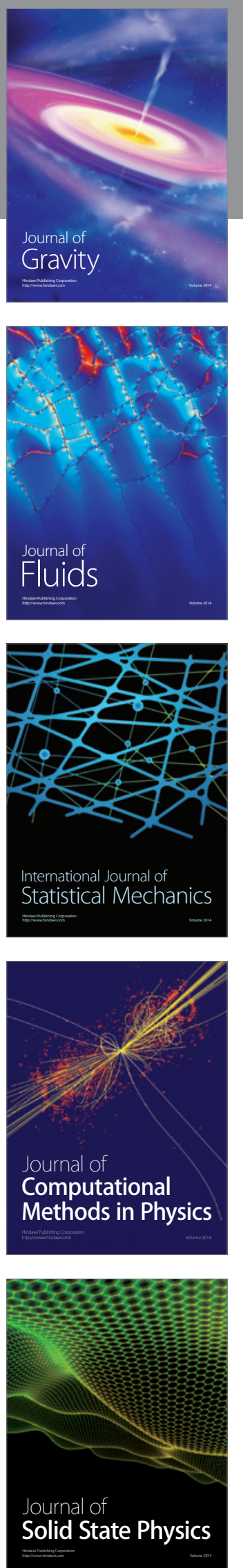
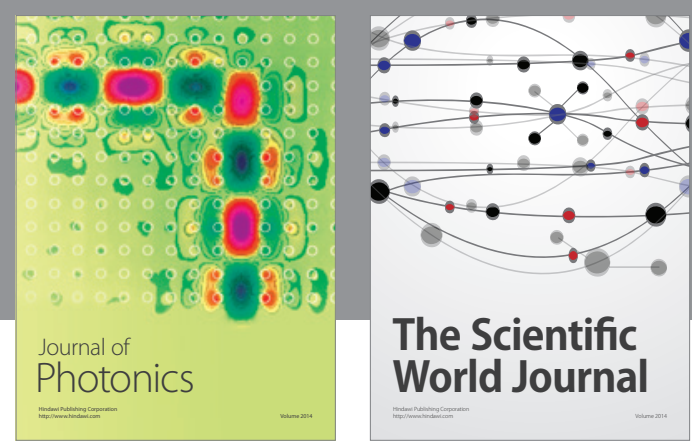

The Scientific World Journal

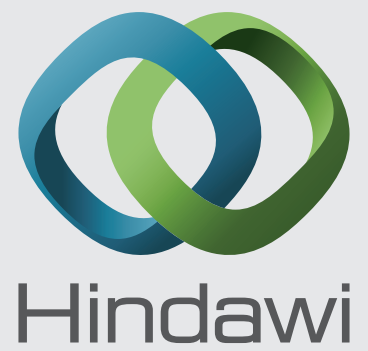

Submit your manuscripts at http://www.hindawi.com
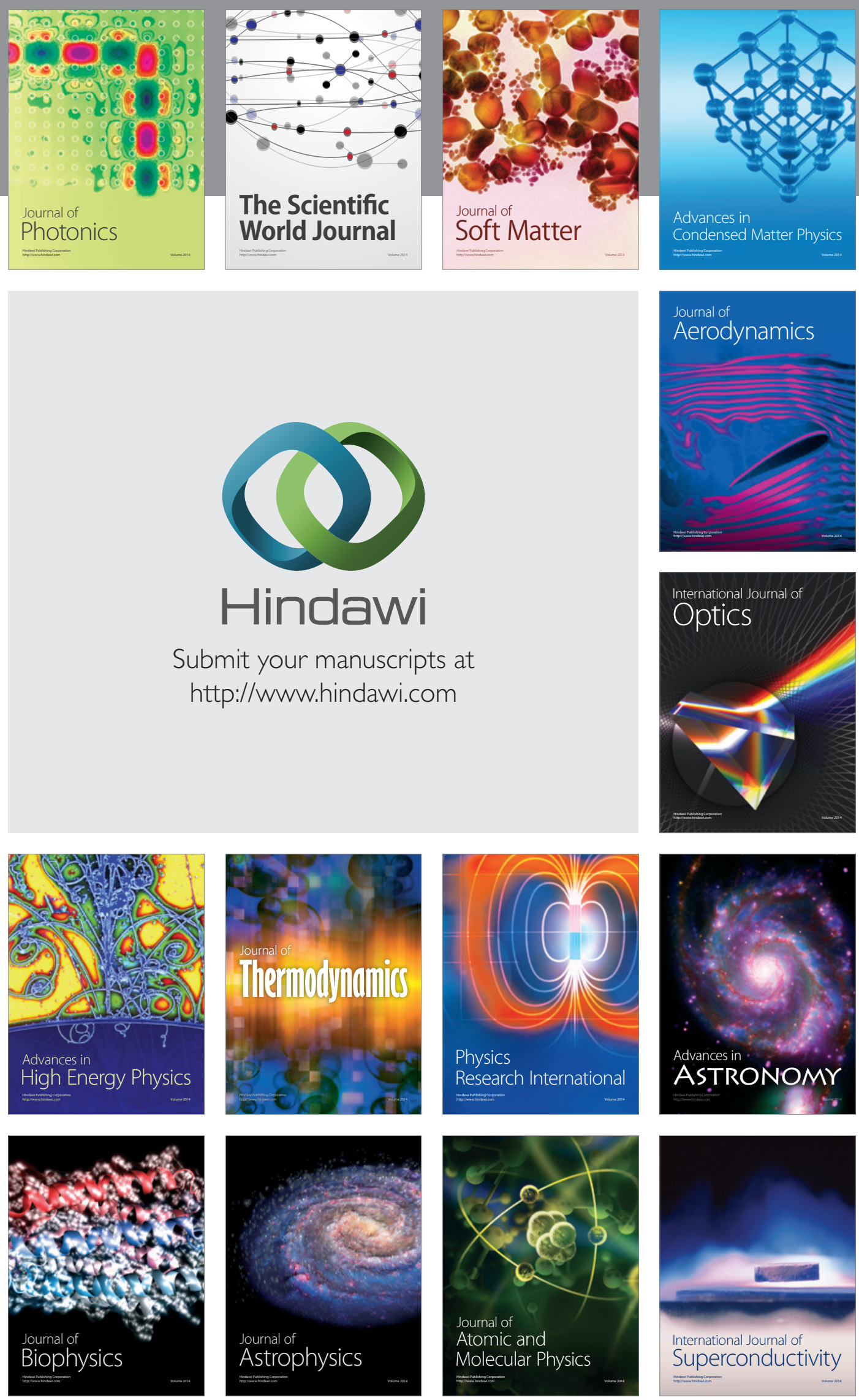
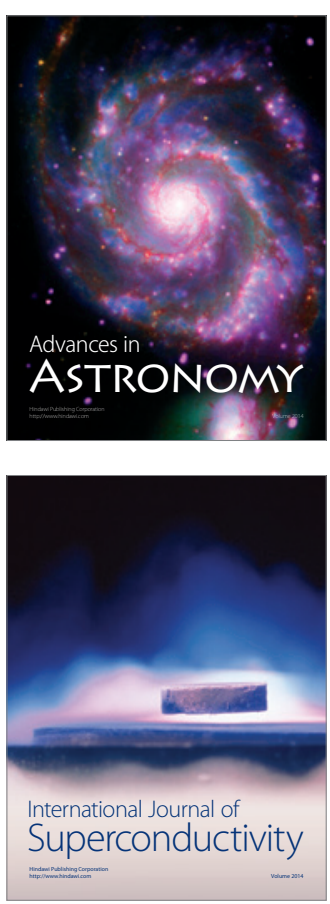